\title{
Cost of Having the Largest Mitochondrial Genome: Evolutionary Mechanism of Plant Mitochondrial Genome
}

\author{
Kazuyoshi Kitazaki and Tomohiko Kubo \\ Research Faculty of Agriculture, Hokkaido University, N-9, W-9, Kita-Ku, Sapporo 060-8589, Japan \\ Correspondence should be addressed to Tomohiko Kubo, tomohiko@abs.agr.hokudai.ac.jp
}

Received 27 January 2010; Accepted 8 March 2010

Academic Editor: Johann Greilhuber

Copyright ( $\odot 2010$ K. Kitazaki and T. Kubo. This is an open access article distributed under the Creative Commons Attribution License, which permits unrestricted use, distribution, and reproduction in any medium, provided the original work is properly cited.

\begin{abstract}
The angiosperm mitochondrial genome is the largest and least gene-dense among the eukaryotes, because its intergenic regions are expanded. There seems to be no functional constraint on the size of the intergenic regions; angiosperms maintain the large mitochondrial genome size by a currently unknown mechanism. After a brief description of the angiosperm mitochondrial genome, this review focuses on our current knowledge of the mechanisms that control the maintenance and alteration of the genome. In both processes, the control of homologous recombination is crucial in terms of site and frequency. The copy numbers of various types of mitochondrial DNA molecules may also be controlled, especially during transmission of the mitochondrial genome from one generation to the next. An important characteristic of angiosperm mitochondria is that they contain polypeptides that are translated from open reading frames created as byproducts of genome alteration and that are generally nonfunctional. Such polypeptides have potential to evolve into functional ones responsible for mitochondrially encoded traits such as cytoplasmic male sterility or may be remnants of the former functional polypeptides.
\end{abstract}

\section{Introduction}

The monophyletic origin of mitochondria, which postulates a single endosymbiotic event involving an $\alpha$-proteobacterialike organism and the common cellular ancestor of eukaryotes, remains a widely accepted concept [1]. Yet it has also become evident that the mitochondrial genomes of extant eukaryotes are more diverged than previously thought $[2,3]$. The angiosperm mitochondrial genome is the largest one known to date $[2,3]$, but this is not the only peculiarity that makes it fascinating. According to a comprehensive review of mitochondrial biology [4], the plant mitochondrial genome "has many characteristics that probably make it one of the most interesting genomes to the molecular biologist." These characteristics include its mode of gene expression and its organizational diversity. From the botanic point of view, we would like to add that the study of the mitochondrial genome provides us with a huge amount of information regarding evolution, nuclear-cytoplasmic interactions, and mitochondrially-encoded traits, and this information is invaluable for crop improvement.
Since the early attempts to investigate the organization and diversity of the angiosperm mitochondrial genome by electron microscopy and gel electrophoresis in the 1980s, a great deal of knowledge has been accumulated. These advances were described in our recent reviews $[5,6]$. Therefore, our focus for this review is the recent activities aimed at identifying the mechanisms that maintain mitochondrial genome organization and that generate alterations, some of which associated with specific phenotypes.

\section{General Features of the Angiosperm Mitochondrial Genome}

The number of angiosperm species whose entire mitochondrial genome sequences are available is currently twelve, and the sequences can be found at the NCBI web site (http://www.ncbi.nlm.nih.gov/genomes/GenomesGroup .cgi?taxid=33090\&opt=organelle). In addition, some intraspecific variants of these genomes have been deposited in the public database. These include the NA-type cytoplasm 


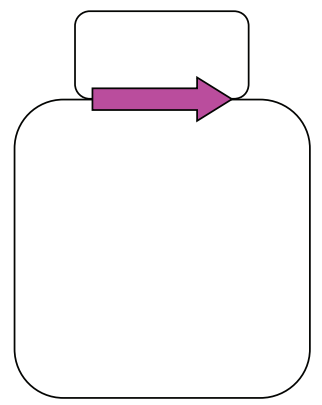

(a)

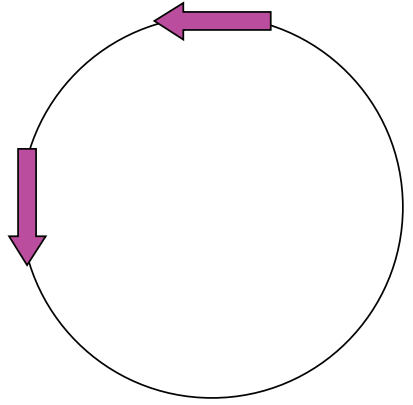

(b)
FIgURE 1: Illustration of a simplified angiosperm mitochondrial genome. (a) Map configuration derived from chromosome walking. Two closed circles share sequence at the region shown by the arrow. (b) Dissolution of the figure 8-like map to create the master circle. The arrows indicate the duplicated sequences introduced into the master circle.

(DDBJ/EMBL/GenBank accession number DQ490952), the T (Texas)-type cytoplasm (DQ490953), the S (USDA)-type cytoplasm (DQ490951), and the C (Charrua)-type cytoplasm (DQ645536) of maize (Zea mays ssp. mays), and the Owen-type cytoplasm (BA000024) of sugar beet (Beta vulgaris). There is no doubt that the number of available mitochondrial sequences will increase rapidly due to improvements in DNA sequencing technology, such as nextgeneration sequencing.

The "genome size" of the angiosperm mitochondrion refers to the size of the "master circle," which is a presumptive circular molecule consisting of all the DNA sequences present at substantial stoichiometry in the mitochondrion. Master circles have usually been constructed by chromosome walking, and fragmented mitochondrial (mt) DNA sequences are linked together based on sequence homologies at the ends of the fragments. However, the resultant maps are not single circles but complex, entangled structures. To help the reader understand this, a simplified map is illustrated in Figure 1, where two closed circles form a figure 8-like structure. This is not due to a cloning artifact such as a chimeric clone. It occurs because the two mtDNA fragments are partly colinear but have diverged from one another. The maps were interpreted as equivalent to the master circles having large duplications, which sometimes reach up to $100 \mathrm{~kb}$. Therefore, the net sequence complexity may be smaller than the size of the master circle. For example, the sizes of the five variant master circles of maize range from 536 to $740 \mathrm{~kb}$, but after omitting the repeated sequences, the complexity ranges from 507 to $537 \mathrm{~kb}$ [7]. Although the entire nucleotide sequence remains unavailable, the mitochondrial genome of muskmelon is estimated to be $2400 \mathrm{~kb}$ [8], which is about ten times larger than that of rapeseed $(222 \mathrm{~kb})$. It should be noted that the presence of the master circle is supported only by molecular cloning, and controversial data have been obtained from other methods including direct visualization of mtDNA molecules and gel electrophoresis of intact mtDNAs [9].
Two DNA species are excluded from the master circle; one includes the plasmid-like DNA molecules of either linear or circular form, which are independent of the master circle in terms of their replication and mode of inheritance [10]. The other group consists of the substoichiometric DNA molecules that exhibit different sequence from the major DNA molecules, the so-called sublimons. The sublimon is clearly distinguished from the major DNA molecule in terms of its maintenance and gene expression. The sublimons may be products of illegitimate recombination or maintained by an unknown mechanism. We will detail more about the sublimons in Section 4.

There are five general features that are characteristic of angiosperm mitochondrial genomes. First, the mitochondrial genome consists of a mixture of DNA species. This was first realized as a result of electron microscopicand gel electrophoresis studies of mtDNA. In addition, as we mentioned above, the maps obtained by chromosome walking were very complex. To explain these observations, it was proposed that the master circle and its derivative circles coexist in the mitochondrion. The mechanism for the generation of subcircles was first proposed by Palmer and Shields [11]. The subcircles are generated via frequent homologous recombination within large (more than $1 \mathrm{~kb}$ ) direct repeats in the master circle (i.e., the smaller circles "loop out" from the master circle). Most of the angiosperm mitochondrial genomes contain one or more sets of such direct repeats that are active in recombination. An exception is documented for black mustard (Brassica hirta) [12]. Also, details of repeated sequences were not mentioned in the first sequence report for the grapevine mitochondrial genome [13], and we failed to find repeated sequences longer than $1 \mathrm{~kb}$ in the DDBJ/EMBL/GenBank sequence entry FM179380 (our unpublished observation). Besides direct repeats, some mitochondrial genomes also contain repeats arranged in inverted orientations. In this case, smaller subcircles are not generated, but isomeric circles that differ from the parental circle by segmental inversion can appear. Due to the presence of these subcircles and isomeric circles, the angiosperm mitochondrial genome is considered to have a multipartite organization. It should be noted that the term multipartite organization is different from heteroplasmy because the isomeric circles and subcircles are the alternative form of the master circle. On the other hand, heteroplasmy in angiosperm mitochondria is caused by sublimons, which are present at low stoichiometry and not entirely colinear with the master circle or any other subcircles.

The molecular basis of homologous recombination in angiosperm mitochondria was mainly determined using DNA cloning and/or DNA gel blot hybridization. For example, nine different genomic products were expected if the three-member repeated sequences on the master circle recombined in petunia $\left(3^{2}=9\right)$ [14]. When the repeated sequence was used as a probe, the expected nine signal bands appeared on a DNA gel blot containing petunia mtDNA. The relative signal intensities corresponding to the recombinant DNA molecules were comparable to those of the parental DNA molecule, indicating that the stoichiometry of the parental and recombinant molecules was similar. 
Such experiments indicate that active recombination occurs within mitochondrial repeated sequences, and recently, biochemical evidence of DNA recombination was obtained [15].

The second feature of the angiosperm mitochondrial genome is that it contains sequences not descended from the ancestral mitochondrial genome but apparently acquired from other genetic compartments, such as plastids, the nucleus, and sexually incompatible organisms (i.e., horizontal transfer). The acquisition of such alien sequences has not been discovered in the mitochondrial genomes of other organisms, apart from those of gymnosperms, which contain plastid-like sequences [16].

The third and fourth features are seen in the gene expression system. The third feature is that most angiosperm mitochondrial genes require the posttranscriptional conversion of specific cytidine residues to uridines, in a process called RNA editing [17]. This C-to-U editing is prevalent, while $\mathrm{U}$-to-C editing is very rare. The fourth feature is the presence of group II introns in up to 10 angiosperm mitochondrial genes [18]. The only group I intron is found in the coxl gene of diverse plant species and was horizontally transferred from fungi. Among the genes containing group II introns, nad1, nad2, and nad5 exhibit trans-splicing, where the exons are transcribed as separate transcripts and then spliced into one mature transcript.

The most striking feature of the angiosperm mitochondrial genome is that there are fewer genes than expected based on the genome size, indicating that it is the least genedense mitochondrial genome [19]. Despite the wide range of genome sizes (222 to $773 \mathrm{~kb}$ among the fully sequenced genomes), the numbers of genes in angiosperm mitochondria (about 50 to 60) do not vary greatly. Angiosperm mitochondria contain more genes than those of humans (37 genes in a $16.6 \mathrm{~kb}$ genome) but less than those of the liverwort Marchantia polymorpha (71 genes in $186 \mathrm{~kb}$ ) and the moss Physcomitrella patens (67 genes in $105 \mathrm{~kb}$ ) [20]. The mitochondrial genome of the gymnosperm Cycas taitungensis $(415 \mathrm{~kb})$ contains 64 genes [21], which is slightly higher than the highest number found in angiosperm mitochondria. An analysis of land plants indicates that some genes have been lost from the angiosperm mitochondria, but the genome size has not been reduced accordingly. In some cases the lost genes appear to have migrated into the nuclear genome, and their translation products are imported into the mitochondria [22]. In other cases, the gene loss is compensated by paralogues in the nuclear genome. For example, the gene rps13 is not present in the Arabidopsis mitochondrion and no migrated copy occurs in the nuclear genome. However, a nuclear rps13 copy of plastid origin appears to encode a mitochondrial RPS13 polypeptide [23]. The genes for tRNA ${ }^{\mathrm{Leu}}$ and $\mathrm{tRNA}^{\mathrm{Arg}}$ are present in Cycas mitochondria [21] but lost from those of angiosperms, and results from an analysis of potato indicate that tRNA molecules charged with Leu and Arg are imported from the cytosol into the mitochondria [24].

It should be noted that there may be additional genes in some angiosperm mitochondria that have not yet been identified. For example, a ribosomal protein gene rpl10 was recently added to the list of mitochondrial genes in tobacco, grapevine, and papaya but is not present in the mitochondria of Arabidopsis, wheat, maize, and sugar beet $[25,26]$.

\section{What Is the Origin of the Intergenic Regions of the Angiosperm Mitochondrial Genome?}

Sequence information has provided insights into the causes of genomic expansion in angiosperm mitochondria. As mentioned above, the intergenic regions are apparently expanded compared with those of other organisms. It appears that the intergenic regions originated via at least two mechanisms. One is the transfer of sequences from other cellular compartments, and even from other organisms, including sexually incompatible species. The other is the alteration of preexisting sequences. Both of the mechanisms are likely involved in the evolution of angiosperm mitochondrial genome.

Plastid-like sequences constitute $1.6 \%$ to $8.8 \%$ of seed plant mitochondrial genomes $[6,13]$. Most of these sequences reside in the intergenic regions; however, it has been reported that an internal segment of a mitochondrial atp 1 gene has been replaced with a plastid atpA sequence [27]. One of the roles of the plastid-like sequences is to provide tRNA genes for mitochondrial translation [28]. Plastidlike sequences can also function as promoters for mitochondrial genes, as exemplified in the rice nad9 gene [29]. Nuclear-derived sequences are also found in the intergenic regions of angiosperm mitochondria and constitute $0.1 \%$ to $13.4 \%$ of the mitochondrial genome [6]. These nuclear-like sequences are rarely conserved among angiosperm species, and no role for them has been reported to date.

It should be noted that the amounts of DNA that are known to be derived from external sources such as plastids and the nucleus are insufficient to explain the origin of the whole intergenic regions, since more than $60 \%$ of the intergenic regions show no homology with any other sequences. This does not mean that the remaining intergenic regions are unique, because the public database does not yet cover all the extant sequences on Earth. In a recent metagenomic analysis of microbial populations, large amounts of novel sequences derived from unidentified organisms were found [30]. Thus, we cannot exclude the possibility that additional sequences, homologous to those in angiosperm mitochondrial in- tergenic regions, might be found in other organisms. In other words, it is possible that some of the intergenic regions of angiosperm mitochondria may have been horizontally transferred from sexually incompatible species including nonplant organisms. Evidence of horizontal transfer into angiosperm mitochondria is accumulating. For example, viral sequences and a group I intron presumably transferred from fungi have been found $[13,31,32]$. Additionally, the plasmid-like DNA elements in some angiosperm mitochondria may be derived from fungi [10]. The integration of these plasmid-like DNA elements into mitochondrial genomes has often been observed $[7,33,34]$. 
Another source of horizontal transfer is from one plant to another. Some research groups claim that mitochondrial genes in some plants, such as the Amborella nad5 and Actinidia rps11 genes, seem to have been transferred between plant species horizontally, based on phylogenetic anomalies [35]. These reports remind us of transtaxonomic homologies between mitochondrial open reading frames (ORFs) in different plant species. For example, orf79 in rice, which is associated with a mitochondrial mutation termed cytoplasmic male sterility (CMS, see below), has a sequence in the $3^{\prime}$ region that shows no homology with normal rice mitochondria or with those of other cereals including maize and wheat. However, in sorghum, another CMS-associated ORF termed orf107 shares sequence homology with the 3' half of orf79 [36].

Three questions arise when horizontal transfer is considered. The first relates to the identity of the donor organism, and a useful tool for tackling this question is phylogenetic analysis [35]. The second question relates to the mechanisms by which DNA fragments are transferred, and the third question refers to the frequency of horizontal transfer. There is some controversy over the frequency of its occurrence in the evolution of angiosperm mitochondrial genomes [13, 32]. This is an important question to be resolved before we can estimate the amount of intergenic sequence that can be explained by horizontal transfer.

It is possible that the intergenic regions of angiosperm mitochondria have accumulated extensive sequence alterations due to duplications, inversions, insertions, and deletions, to the point that the original sources of some sequences cannot be identified by homology searches. Homologous recombination has occurred quite frequently during the evolution of angiosperm mitochondria. When the intergenic regions are investigated in detail, short (less than $25 \mathrm{bp}$ ) stretches of mitochondrial genes or other known sequences are occasionally found [37]. These might be remnants of extensive rearrangements. The mitochondrial genome of cucumber may be a good example of genome expansion, because it has the second largest mitochondrial genome identified to date ( $>1500 \mathrm{~kb}$ [38], of which $136 \mathrm{~kb}$ has been sequenced [39]). An analysis of the obtained sequences revealed that $15 \%$ of the region consists of repeat sequences that can be grouped into seven families, indicating that expansion of the genome can, at least in part, be explained by duplication.

\section{How Has the Angiosperm Mitochondrial Genome Evolved?}

In general, sequence conservation among the intergenic regions of angiosperm mitochondrial genomes is too low to support any strong functional constraints on these regions (although some sequence similarities have been found within $\sim 100$ bp upstream of some angiosperm mitochondrial genes [40]). Moreover, intergenic regions may contain mitotypespecific sequences that are not shared by other mitochondrial genomes. It is known that the rate of nucleotide substitution in angiosperm mitochondrial genomes is very low compared with other organisms, although the rate is occasionally accelerated in some specific plant lineages [41, 42]. On the other hand, one can easily find alterations in the arrangement of mitochondrial genes, even between closely related species [7]. Therefore, a gene cluster that is conserved in algal or moss mitochondrial genomes [20] is rarely seen in angiosperm mitochondria. In summary, molecular diversity in angiosperm mitochondrial genomes involves the occurrence of mitotype-specific sequences and genome rearrangements. The occurrence of mitotype-specific sequences seems to be due to the diverse origins of sequences in the intergenic regions, which we discussed in the previous section.

The primary mechanism of genome rearrangement seems to be homologous recombination between repeated sequences. As mentioned earlier, most angiosperm mitochondrial genomes contain recombination-active repeat sequences of over $1 \mathrm{~kb}$, and recombination occurs regularly via these repeats. But these are insufficient to explain all the genome rearrangements that occur. It appears that homologous recombination leading to genome rearrangements involves short (less than $1 \mathrm{~kb}$ ) repeat sequences that are usually inactive in recombination. Circumstantial evidence for the association between rare recombination events and these short repeat sequences is provided by comparative analyses of related genomes [7, 37, 43, 44]. One can find the short repeat sequences at the junctions between rearranged DNA fragments. These recombination events not only explain the changes in genomic arrangements but are also associated with deletions and duplications [45]. An interesting observation is the lack of repeats within specific size ranges in Arabidopsis where there are no repeat sequences between $557 \mathrm{bp}$ and $4.3 \mathrm{~kb}$ in length, although there are 38 repeat sequence families that are either shorter or longer than this size range [46]. The two largest repeat sequences (4.3 and $6.6 \mathrm{~kb}$ ) are active in recombination while those that are shorter than 557 bp are reported as inactive [47].

If recombination occurred freely in mitochondria, it would result in the impairment of genomic function. Indeed, mitochondrial mutants exhibiting respiratory impairments have occurred as a result of deletions accompanied by losses of mitochondrial genes, through homologous recombination at short repeat sequences. Examples include the maize nonchromosomal stripes mutant, the cucumber mosaic mutant, tobacco CMS I and CMS II, and the Arabidopsis maternally distorted leaves mutant [48-51]. In order to preserve mitochondrial functioning there must be a mechanism to suppress free recombination and/or the amplification of irregularly recombined DNA molecules. Nuclear genes whose lesions result in increased amounts of irregularly recombined mitochondrial DNA molecules have been reported. These include Msh1, RecA3, and OSB1 [52-54]. The Arabidopsis mutant lacking Msh1 function has been investigated in detail [46]. In these plants there is an increase in recombination via short repeat sequences ranging from 108 to $556 \mathrm{bp}$, but shorter repeats of less than $108 \mathrm{bp}$ do not respond. This suggests that another gene may suppress recombination via the very short repeat sequences. Consistent with this, the disruption of RecA1 
in Physcomitrella results in the activation of mitochondrial genome recombinations involving repeats of less than $100 \mathrm{bp}$ [55]. In the presence of functional Mshl, recombination via short repeats is apparently suppressed, but recombinant DNA molecules are not completely absent; they can be present at very low stoichiometric levels as sublimons. It is evident that Msh 1 also plays a similar role in tobacco and tomato, because knockdown experiments involving the Msh1 orthologues in these plants result in novel mitochondrial genotypes [56]. Although the genes responsible have not been identified, plant lines that generate multiple mitochondrial genotypes in their offspring are known. These include maize $\mathrm{P} 2$ and cucumber line B $[49,57]$.

We know empirically that the organization of the mitochondrial genome is usually unaltered over many generations. However, on an evolutionary scale recombination via short repeats has occurred, and such mtDNA has become dominant. Cumulative data indicate an association between mitochondrial diversity and heteroplasmy. A long-standing hypothesis, that an alteration of mtDNA is first carried as a sublimon and then amplified to the level of a major DNA molecule $[58,59]$, is still attractive and remains to be explored. In the common bean, CMS-type mtDNA lurks as a sublimon in the ancestral, fertile strain [60]. In Arabidopsis, the major mtDNAs of the three ecotypes C24, Col-0, and Ler are organizationally different from each other; however each ecotype carries the two noncognate mtDNAs as sublimons $[46,61]$. Therefore, recombinant DNA molecules involved in genomic diversity can occur as sublimons. Because sublimons are apparently transcriptionally inactive $[62,63]$, mutations may easily accumulate in them.

In order to accumulate genomic alterations in sublimons, the heteroplasmic state should be transmitted from one generation to the next. However, in animal mitochondria, heteroplasmy is resolved into homoplasmy during oogenesis [64]. There is some controversy regarding this process. It is not clear whether the copy number of the mtDNA is decreased to exert a bottleneck effect resulting in homoplasmy, or whether the copy number is not reduced but the number of effective mtDNA segregation units is small [64]. However, there has been no such study in angiosperms.

How sublimons are maintained in angiosperm mitochondria is a very important question [65]. In the common bean, sublimons are classified into two classes based on quantitative data [66]. One class is present at a ratio of approximately $10^{-3}: 1$ relative to the major DNA molecule and can be detected rather easily by DNA gel blot hybridization or PCR. The other class occurs at a ratio of approximately $10^{-6}: 1$, and highly sensitive methods are needed for detection of these molecules. The sublimons may be generated via occasional recombination at short repeat sequences, and/or they may replicate inefficiently, so that the copy number is reduced compared with that of the major DNA molecule. The latter possibility assumes an additional mechanism to ensure the transmission of the sublimon to the next generation; however the existence of such a mechanism remains obscure. It should be noted that paternal leakage, that is, mitochondrial transmission via pollen, has been suggested as one of the sources of sublimons $[67,68]$. Most angiosperm species show exclusively maternal inheritance of the mitochondrial genome. However, paternal leakage has been documented in several cases [67] and may contribute to heteroplasmy [68].

The altered sublimon needs to be amplified to the level of the major mtDNA molecule in order to manifest as a genomic alteration. Such increases in the copy numbers of sublimons can occur as a result of mutations of nuclear genes or physiological stress. This phenomenon, together with a rapid decrease in the copy number of the mtDNA molecule to a substoichiometric level, is called substoichiometric shifting (SSS). SSS was first recognized in common bean, where the introduction of the nuclear gene Fr into a plant by crossing resulted in a decrease in a specific class of mitochondrial DNA molecules in the subsequent generation [62]. The Msh1 gene (mentioned above) was first identified as a mutant allele causing SSS in Arabidopsis [52], suggesting that the genes that suppress homologous recombination may also help to regulate the copy numbers of recombinant DNA molecules.

The SSS-like phenomenon can also be observed in the absence of any mutations. A well-known example is tissue culture, which can cause increases or decreases in the copy numbers of specific mtDNA molecules [69]. This suggests that SSS may occur as a result of developmental triggers. The tissues or organs where SSS might occur are the meristem and the egg, where the "transmitted form" of the mtDNA, containing all components of the genome including sublimons, is expected $[60,70]$.

\section{Why Do Most of the Unique ORFs Not Matter in Angiosperm Mitochondria?}

Two classes of mitochondrial mutants are known in angiosperms. One is associated with lesions in indispensable mitochondrial genes. These can be caused by deletions or (rarely) point mutations $[71,72]$. In the other class, no deleterious mutation can be found in any of the genes; nevertheless a specific mutant polypeptide is translated in the mitochondria. When the ORF encoding the polypeptide is cloned, it is structurally distinct from the standard mitochondrial gene and consists of segments of known or unknown sequences. Therefore, in this class of mitochondrial mutants, a unique ORF could be associated with the phenotype. CMS, which shows a phenotype of pollen abortion in plants that are otherwise developmentally normal, is one representative of this class of mutations. CMS has been reported in more than 140 plant species [36], and the number of identified genes associated with the phenotype has reached 23, of which 21 involve unique ORFs [73]. An example is the maize urf13$T$ gene, which contains two duplicated segments of rrn26, forming an ORF that encodes 115 amino acid residues [74]. A second example is the petunia $p c f$ gene, which contains a duplicated segment of atp9, two duplicated segments of $\operatorname{cox} 2$, and a sequence of unknown origin, forming an ORF that encodes 402 amino acid residues [75]. There is no sequence homology between any of the known CMS-associated ORFs except in two cases: orf224, associated with Brassica pol 
CMS, and orf222, associated with Brassica nap CMS, exhibit $79 \%$ homology at the amino acid level [36]; and rice orf79 and sorghum orf107 show some similarity. Mitochondrially encoded disease susceptibility is another phenotype that is associated with unique ORFs: the maize urf13-T causes not only CMS but also susceptibility to a toxin produced by the pathogen Cochliobolus heterostrophus [36]. The rough lemon gene Acrs (containing a plastid-like sequence) is responsible for sensitivity to a toxin produced by the fungus Alternaria alternata [76]. Because the urf13-T sequence can be deleted by tissue culture to obtain male fertile and toxininsensitive plants, the $u r f 13-T$ gene is dispensable for normal mitochondrial function [36].

In addition to these unique ORFs that cause mutant phenotypes, many more unique ORFs, which cause no obvious phenotypes, are present in angiosperm mitochondrial genomes. For example, Arabidopsis mitochondria contain unique ORFs with duplicated segments derived from known mitochondrial genes and sequences of unknown origin [77], but no mitochondrially encoded phenotypes such as male sterility or disease susceptibility are known in this ecotype. Therefore, most unique ORFs seem to cause no problems for the plants. The large coding capacity of the angiosperm mitochondrial genome and its structural fluidity appear to have resulted in the frequent emergence and retention of unique ORFs during the course of plant evolution. Why are so many unique ORFs tolerated? To address this question, we would like to discuss the features that distinguish the CMSassociated ORFs from other unique ORFs.

As reviewed by Holec et al. [78], the multiplicity of promoter sequences and the absence of efficient transcription termination mechanisms lead to the transcription not only of mitochondrial genes but also of intergenic regions. Thus, transcription may occur anywhere in the mitochondrial genome, including regions containing unique ORFs. However, most illegitimate transcripts are degraded by a mechanism involving a $3^{\prime}$ to $5^{\prime}$ exonuclease called mitochondrial polynucleotide phosphorylase, because they are not associated with stabilizing factors that regulate the abundance of mitochondrial RNA. Cotranscription with functional mitochondrial genes is one way for a unique ORF to be transcribed and to accumulate as a stable RNA, because the stabilizing factors for the mitochondrial gene may incidentally protect the adjacent unique ORF. In fact, many CMS associated genes are cotranscribed with downstream or upstream mitochondrial genes [79]. The significance of this arrangement is evident from the observation that the restoration of fertility often accompanies the cleaving off of the unique ORF-coding sequence from the polycistronic transcript (see an example for rice in [80]). On the other hand, it should be noted that cotranscription does not always result in the translation of the unique ORF. Two ORFs in sugar beet mitochondria, orf119 (consisting of a duplicated segment of nad9 and 340 bp of unknown origin) and orf324 (containing a duplicated segment of atp 8 and 751 bp of unknown origin), are transcribed along with the downstream genes atp1 and rps13, respectively. However, the ORF119 and ORF324 antisera failed to react with any mitochondrial proteins, and no band corresponding to such polypeptides was observed among the radiolabelled in organello translation products [81-83]. A possible explanation is that the unique transcripts lack cis regulatory elements required for translation. However, sugar beet orf324, like the CMS-associated orf522 of sunflower and orf 224 and orf 222 of Brassica, contains a duplicated segment of atp 8 in its $5^{\prime}$ region. In sugar beet, atp 8 and orf324 share $321 \mathrm{bp}$ of $5^{\prime}$ upstream sequence and $59 \mathrm{bp}$ of sequence that encodes the amino terminal part of the protein (Figure $2(\mathrm{a})$ ). It is possible that orf324 is translated, but the translation products are not detected due to protein degradation.

The translation of a unique ORF is inevitable if it fused in frame with a genuine mitochondrial gene. This situation has occasionally occurred during the course of evolution. Some examples of such unique ORFs, whose translation products have been analyzed, are listed in Table 1 . In each case, the origin of the fused sequence is not completely clear. It is intriguing that both the $\mathrm{N}$-terminal and C-terminal regions of mitochondrial genes can be targets of genomic alteration, but not all result in mitochondrial impairment. Of the nine genes listed in Table 1, only two are associated with CMS. In the cases where the fused polypeptide is cleaved (7 genes), the peptide associated with the unique ORF could be detected in two cases. The mechanism by which the core region is cleaved off is not fully understood, because we currently lack sufficient knowledge of posttranslational process in angiosperm mitochondria.

A well-known example of this situation is atp6, which consists of a conserved C-terminal region of 249 to 252 amino acid residues (core region) and a diverged $\mathrm{N}$-terminal region of 5 to 389 residues, depending on the mitotype (the N-terminal extension) (Figure 2(b)). Because the standard translation initiation codon (Met) is at the amino-terminus of the extension and the core region starts with a Ser residue, translation of the core region must follow the translation of the N-terminal extension. From the fused polypeptide, the core region is yielded as a mature ATP6 polypeptide after cleavage of the N-terminal extension at a site just before the consensus motif Ser-Pro-Leu. This motif is the target site of an endopeptidase, presumably the orthologue of the yeast ATP23 [91, 92] (see below). There is no evidence that the cleaved $\mathrm{N}$-terminal extension is accumulated as a solitary polypeptide, apart from one exception: sugar beet atp6, whose N-terminal extension is associated with CMS and known as preSatp6 [83]. In cereal mitochondria, the gene rps2 encodes a fused C-terminal extension [93] that is not encoded in the Marchantia, Physcomitrella, or Cycas mitochondria. The fused rps2 of maize is translated, then the C-terminal extension is cleaved off, but it can be detected as a solitary polypeptide [86]. Sugar beet has a unique $\mathrm{cm} C$ gene in which the conserved translational initiation codon is lost, but instead, the chimeric N-terminal extension consists of a duplicated segment of atp 9 and a sequence of unknown origin [90] (Figure 2(b)). The mature CCMC polypeptide is yielded from the fused polypeptide by cleavage of the N-terminal extension [90]. It has been suggested that the putative cleavage site may precede SerPro-Leu, by analogy with atp6. The solitary N-terminal extension has not been detected. To date, no phenotypes 


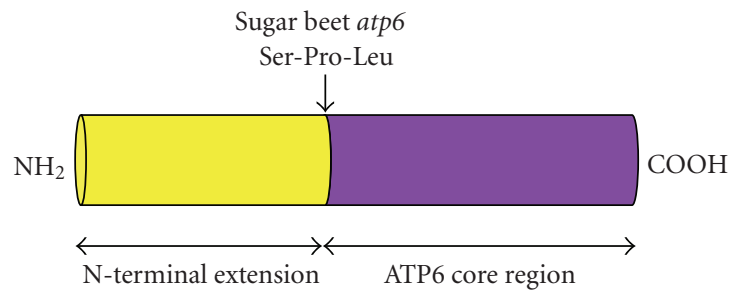

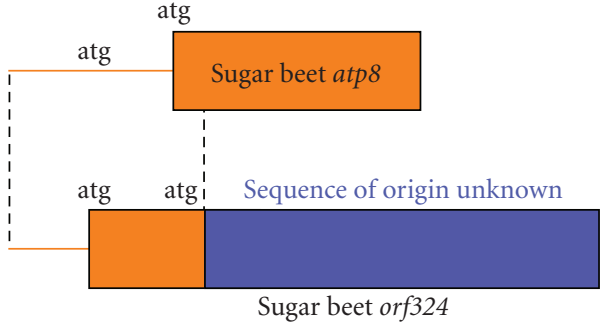

(a)

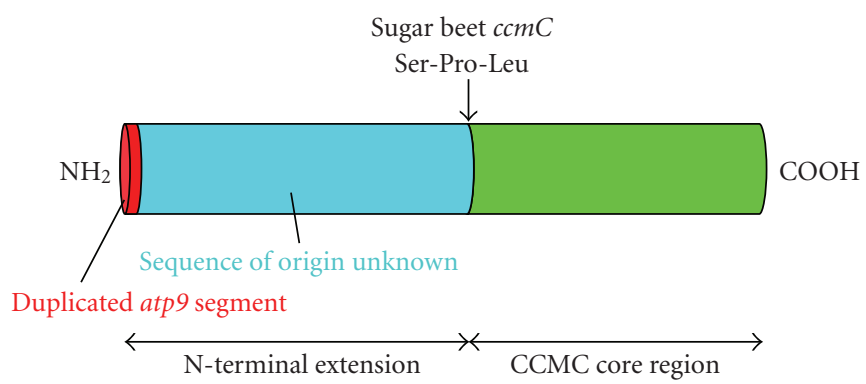

(b)

FIGURE 2: Organizational relationships between genuine mitochondrial genes and unique ORFs at the DNA (a) and polypeptide (b) levels. (a) Comparison of sugar beet atp8 and orf324. Open reading frames are boxed. Dashed lines indicate the boundaries of shared sequences. Two in-frame initiation codons (atg) are shown, of which the second is conserved among angiosperm atp 8 genes. Sugar beet orf324 contains a duplicated segment of atp 8 and a sequence of unknown origin. (b) Comparison of polypeptides deduced from the sugar beet atp6 and the sugar beet $\mathrm{ccmC}$. The N-terminal extensions and the core regions are indicated. The putative cleavage motif is Ser-Pro-Leu. The N-terminal extension of sugar beet $\mathrm{ccm} C$ consists of a duplicated segment of atp 9 and a sequence of unknown origin.

have been associated with the maize rps2 or the sugar beet $\mathrm{ccm} C$.

It is not known whether there are any roles for these extensions in angiosperm mitochondria, or whether the fusion proteins have acquired novel functions, apart from the exception of the sugar beet atp6 [83]. No extensions have been found in mammalian atp6 genes; however an $\mathrm{N}$-terminal extension (10 amino acid residues) of atp6 is required for the efficient assembly of the mature ATP6 polypeptide into the ATP9 ring in Saccharomyces cerevisiae [94]. In this process, a dual-function metalloprotease, ATP23, cleaves the N-terminal extension off and, together with the putative molecular chaperon ATP10, assembles the mature ATP6 into the $\mathrm{F}_{0}$ ATPase [91, 92]. It should be noted that potential homologues of atp 23 and atp 10 are also encoded in the Arabidopsis nuclear genome as at $3 \mathrm{~g} 03420$ and at 1 008220, respectively (our unpublished observation).

It is possible that the translational products of unique ORFs, if any, are subjected to degradation by mitochondrial quality control systems such as AAA- and Lon proteases. These proteases are indeed encoded in Arabidopsis and have been shown to participate in protein degradation [95-97]. The mitochondrial protein degradation system is also associated with the anther-specific accumulation of the CMS-associated ORF239 polypeptide in the common bean [98]. The petunia CMS-associated PCF protein is trimmed into a smaller polypeptide from a precursor [75]. Therefore, it seems likely that most unique polypeptides are degraded. However, some of the cleaved polypeptides derived from unique ORFs (e.g., CMS-associated ORFs and the maize rps $2 A$ extension) might be tough targets for the protein degradation system due to their higher-order structures. For example, if a polypeptide were embedded in the mitochondrial membrane and its extracellular domain were inaccessible to molecular chaperones, this would prevent it from being pulled out of the membrane for degradation [99]. In rice, ORF79 is a stable membrane-integrated polypeptide expressed in all tissues. In a line of CMS rice carrying a variant of orf79, termed L-orf79, the L-ORF79 polypeptide is not detected [100]. Itabashi et al. [100] suggested that this might be due to a sequence alteration in the $5^{\prime}$ untranslated region that prevents translation. However, it is also possible that a Glu to Ala substitution in the coding region may alter the higher-order structure, making L-ORF79 accessible to the degradation system. A variant of atp6 in sugar beet termed preS-3atp6, whose encoded amino acid sequence shows $88 \%$ homology with that of preSatp6, is translated and the corresponding polypeptide is detected in leaves and roots but not anthers. This suggests that the polypeptide may be accessible to a tissue-specific degradation system, unlike preSATP6, which is quite stable $[83,101]$.

Overall, unique ORFs do not create problems for plants as long as their translation products are controlled by posttranscriptional and posttranslational processes. In addition, it appears that some nontoxic or nonfunctional polypeptides derived from unique ORFs (e.g., see Table 1) can exist in angiosperm mitochondria. This suggests that the accumulation of unique polypeptides per se is insufficient to cause mitochondrially encoded phenotypes. In other words, the CMS- or disease susceptibility-associated polypeptides may have particular characteristics that enable them to modulate mitochondrial function, leading to specific phenotypes. 
TABLE 1: Unique ORFs that are fused with genuine mitochondrial genes.

\begin{tabular}{|c|c|c|c|c|c|c|}
\hline Name of gene & $\begin{array}{l}\text { Position of fused } \\
\text { unique ORF }\end{array}$ & $\begin{array}{l}\text { Detection of fused } \\
\text { polypeptide }\end{array}$ & $\begin{array}{l}\text { Detection of cleaved } \\
\text { core region }^{2}\end{array}$ & $\begin{array}{l}\text { Detection of cleaved } \\
\text { extension }^{3}\end{array}$ & Note & References \\
\hline Carrot atp 8 & $\mathrm{C}(57)$ & Yes & Yes $^{4}$ & No & & {$[84]$} \\
\hline Carrot $\operatorname{cox} 1$ & $\mathrm{C}(224,284)$ & No & Yes & ND & & {$[85]$} \\
\hline Maize $r p s 2 A$ & $\mathrm{C}(330)$ & Yes & Yes & Yes & & {$[86]$} \\
\hline $\begin{array}{l}\text { Sugar beet } \\
\text { Gnad9-1 }\end{array}$ & $\mathrm{C}(14)$ & Yes & No & $\mathrm{ND}^{5}$ & $\begin{array}{l}\text { Complex I exhibits } \\
\text { normal activity. }\end{array}$ & {$[71]$} \\
\hline Sorghum coxl & $C(101)$ & Yes & No & $\mathrm{ND}^{5}$ & $\begin{array}{l}\text { Presumably } \\
\text { associated with CMS. }\end{array}$ & {$[87]$} \\
\hline Petunia atp6 & $\mathrm{N}(145)$ & $\mathrm{No}^{6}$ & Yes & $\mathrm{ND}^{5}$ & & {$[88]$} \\
\hline $\begin{array}{l}\text { Radish Ogura } \\
\text { atp6 }\end{array}$ & $\mathrm{N}(174)$ & $\mathrm{No}^{6}$ & Yes & $\mathrm{ND}^{5}$ & & [89] \\
\hline Sugar beet atp6 & $\mathrm{N}(387)$ & $\mathrm{No}^{6}$ & Yes & Yes & $\begin{array}{l}\text { Cleaved extension } \\
\text { forms oligomer. } \\
\text { Presumably } \\
\text { associated with CMS. }\end{array}$ & {$[83]$} \\
\hline Sugar beet $\mathrm{ccm} C$ & $\mathrm{~N}(277)$ & Yes & Yes & No & & {$[90]$} \\
\hline
\end{tabular}

The identification of such "elite" unique polypeptides is of practical importance. A transgenic approach sometimes (but not always) works well, wherein plants expressing specific unique ORFs fused with mitochondrial import signal peptides express male sterility or toxin sensitivity [79, 101105]. Microorganisms such as E coli can also be used as hosts to examine the toxin sensitivities conferred by specific ORFs $[36,76]$. It is known that the polypeptides encoded by maize urf13-T, radish orf138 (associated with CMS), and sugar beet preSatp6 form oligomers in the inner membrane $[36,83$, 106], which may function as ionophores [107]. However, sugar beet ORF129, which also causes CMS when targeted to the mitochondria, shows a different localization pattern [101]. Thus, there appear to be multiple ways by which unique polypeptides can modulate mitochondrial function.

\section{Concluding Remarks and Perspectives}

The angiosperm mitochondrial genome is the largest one known to date and is highly diverged in terms of genome size, gene arrangement, and sequences in the intergenic regions. The mechanisms by which angiosperm mitochondrial genomes have diverged may include horizontal transfer from other organisms, although the extent of this contribution remains to be evaluated. The recombination of mtDNA should be under the strict control to maintain genomic integrity. In leaves and other vegetative tissues that have been used for mtDNA analyses, two contradictory activities have been found to coexist. One seems to promote recombination within repeats of over $1 \mathrm{~kb}$, and the other suppresses recombination between repeats of less than $1 \mathrm{~kb}$. The existence of heteroplasmy in angiosperm mitochondria provides us with a unique opportunity for exploration in the field of cytoplasmic genetics, because the situation in plants appears to be different from that in mammals. One of the major questions to be resolved is the maintenance and transmission of sublimons. It might be necessary to examine the mitochondrial genome organization in the meristem and the egg, both of which are difficult to isolate and examine. The extent of paternal leakage of the mitochondrial genome might be underestimated, since this is a likely cause of heteroplasmy and horizontal transfer. Because the functional constraints of the intergenic regions are low, and because the angiosperm mitochondrial genome undergoes frequent rearrangements on an evolutionary scale, unique ORFs are common. Fused mitochondrial genes provide appropriate examples to examine how the expression of unique ORFs is controlled. Posttranslational control mechanisms, including the quality control system, are important for the proper expression of fused mitochondrial genes. It is apparent that useless polypeptides derived from unique ORFs can exist in mitochondria, presumably because they can escape the quality control system. We would like to hypothesize that there exists an evolutionary association between these polypeptides and CMS-associated polypeptides. The apparently nonfunctional polypeptides may have the potential to evolve into causal agents of CMS, or they may be remnants of ancient CMS-causing genes.
Abbreviations
CMS: Cytoplasmic male sterility
ORF: Open reading frame
SSS: Substoichiometric shifting. 


\section{Acknowledgments}

This work was supported in part by Grants in Aid for Scientific Research from the Ministry of Education, Culture, Sports, Science, and Technology and the Program for Promotion of Basic and Applied Research for Innovation in Biooriented Industry (BRAIN).

\section{References}

[1] M. W. Gray, "Evolution of organellar genomes," Current Opinion in Genetics and Development, vol. 9, no. 6, pp. 678687, 1999.

[2] M. W. Gray, B. F. Lang, and G. Burger, "Mitochondria of protists," Annual Review of Genetics, vol. 38, pp. 477-524, 2004.

[3] C. E. Bullerwell and M. W. Gray, "Evolution of the mitochondrial genome: protist connections to animals, fungi and plants," Current Opinion in Microbiology, vol. 7, no. 5, pp. 528-534, 2004.

[4] I. E. Scheffler, Mitochondria, Wiley-Liss, New York, NY, USA, 2nd edition, 2008.

[5] T. Kubo and T. Mikami, "Organization and variation of angiosperm mitochondrial genome," Physiologia Plantarum, vol. 129, no. 1, pp. 6-13, 2007.

[6] T. Kubo and K. J. Newton, "Angiosperm mitochondrial genomes and mutations," Mitochondrion, vol. 8, no. 1, pp. 5$14,2008$.

[7] J. O. Allen, C. M. Fauron, P. Minx, et al., "Comparisons among two fertile and three male-sterile mitochondrial genomes of maize," Genetics, vol. 177, no. 2, pp. 1173-1192, 2007.

[8] B. L. Ward, R. S. Anderson, and A. J. Bendlich, "The mitochondrial genome is large and variable in a family of plants (Cucurbitaceae)," Cell, vol. 25, no. 3, pp. 793-803, 1981.

[9] H. Dai, Y.-S. Lo, A. Litvinchuk, et al., "Structural and functional characterizations of mung bean mitochondrial nucleoids," Nucleic Acids Research, vol. 33, no. 15, pp. 47254739, 2005.

[10] H. Handa, "Linear plasmids in plant mitochondria: peaceful coexistences or malicious invasions?" Mitochondrion, vol. 8, no. 1, pp. 15-25, 2008.

[11] J. D. Palmer and C. R. Shields, "Tripartite structure of the Brassica campestris mitochondrial genome," Nature, vol. 307, no. 5950, pp. 437-440, 1984.

[12] J. D. Palmer and L. A. Herbo, "Unicircular structure of the Brassica hirta mitochondrial genome," Current Genetics, vol. 11, no. 6-7, pp. 565-570, 1987.

[13] V. V. Goremykin, F. Salamini, R. Velasco, and R. Viola, "Mitochondrial DNA of Vitis vinifera and the issue of rampant horizontal gene transfer," Molecular Biology and Evolution, vol. 26, no. 1, pp. 99-110, 2009.

[14] O. Folkerts and M. R. Hanson, "Three copies of a single recombination repeat occur on the $443 \mathrm{~kb}$ master circle of the Petunia hybrida 3704 mitochondrial genome," Nucleic Acids Research, vol. 17, no. 18, pp. 7345-7357, 1989.

[15] M. Manchekar, K. Scissum-Gunn, D. Song, F. Khazi, S. L. McLean, and B. L. Nielsen, "DNA recombination activity in soybean mitochondria," Journal of Molecular Biology, vol. 356, no. 2, pp. 288-299, 2006.

[16] D. Wang, Y.-W. Wu, A. C.-C. Shih, C.-S. Wu, Y.-N. Wang, and S.-M. Chaw, "Transfer of chloroplast genomic DNA to mitochondrial genome occurred at least 300 MYA," Molecular Biology and Evolution, vol. 24, no. 9, pp. 20402048, 2007.

[17] M. Takenaka, D. Verbitskiy, J. A. van der Merwe, A. Zehrmann, and A. Brennicke, "The process of RNA editing in plant mitochondria," Mitochondrion, vol. 8, no. 1, pp. 3546, 2008.

[18] L. Bonen, "Cis- and trans-splicing of group II introns in plant mitochondria," Mitochondrion, vol. 8, no. 1, pp. 26-34, 2008.

[19] M. Unseld, J. R. Marienfeld, P. Brandt, and A. Brennicke, "The mitochondrial genome of Arabidopsis thaliana contains 57 genes in 366,924 nucleotides," Nature Genetics, vol. 15, no. 1, pp. 57-61, 1997.

[20] K. Terasawa, M. Odahara, Y. Kabeya, et al., "The mitochondrial genome of the moss physcomitrella patens sheds new light on mitochondrial evolution in land plants," Molecular Biology and Evolution, vol. 24, no. 3, pp. 699-709, 2007.

[21] S.-M. Chaw, A. C.-C. Shih, D. Wang, Y.-W. Wu, S.-M. Liu, and T.-Y. Chou, "The mitochondrial genome of the gymnosperm Cycas taitungensis contains a novel family of short interspersed elements, Bpu sequences, and abundant RNA editing sites," Molecular Biology and Evolution, vol. 25, no. 3, pp. 603-615, 2008.

[22] K. L. Adams and J. D. Palmer, "Evolution of mitochondrial gene content: gene loss and transfer to the nucleus," Molecular Phylogenetics and Evolution, vol. 29, no. 3, pp. 380-395, 2003.

[23] K. L. Adams, D. O. Daley, J. Whelan, and J. D. Palmer, "Genes for two mitochondrial ribosomal proteins in flowering plants are derived from their chloroplast or cytosolic counterparts," Plant Cell, vol. 14, no. 4, pp. 931-943, 2002.

[24] L. Marechal-Drouard, P. Guillemaut, A. Cosset, et al., "Transfer RNAs of potato (Solanum tuberosum) mitochondria have different genetic origins," Nucleic Acids Research, vol. 18, no. 13, pp. 3689-3696, 1990.

[25] J. P. Mower and L. Bonen, "Ribosomal protein L10 is encoded in the mitochondrial genome of many land plants and green algae," BMC Evolutionary Biology, vol. 9, article 265, 2009.

[26] N. Kubo and S.-I. Arimura, "Discovery of the rpl10 gene in diverse plant mitochondrial genomes and its probable replacement by the nuclear gene for chloroplast RPL10 in two lineages of angiosperms," DNA Research, vol. 17, pp. 1-9, 2010.

[27] W. Hao and J. D. Palmer, "Fine-scale mergers of chloroplast and mitochondrial genes create functional, transcompartmentally chimeric mitochondrial genes," Proceedings of the National Academy of Sciences of the United States of America, vol. 106, no. 39, pp. 16728-16733, 2009.

[28] L. Maréchal-Drouard, J. H. Weil, and A. Dietrich, "Transfer RNAs and transfer RNA genes in plants," Annual Review of Plant Physiology and Plant Molecular Biology, vol. 44, no. 1, pp. 13-32, 1993.

[29] M. Nakazono, S. Nishiwaki, N. Tsutsumi, and A. Hirai, "A chloroplast-derived sequence is utilized as a source of promoter sequences for the gene for subunit 9 of $\mathrm{NADH}$ dehydrogenase (nad9) in rice mitochondria," Molecular and General Genetics, vol. 252, no. 4, pp. 371-378, 1996.

[30] O. Beja, M. T. Suzuki, E. V. Koonin, et al., "Construction and analysis of bacterial artificial chromosome libraries from a marine microbial assemblage," Environmental Microbiology, vol. 2 , no. 5, pp. 516-529, 2000. 
[31] J. Marienfeld, M. Unseld, and A. Brennicke, "The mitochondrial genome of Arabidopsis is composed of both native and immigrant information," Trends in Plant Science, vol. 4, no. 12, pp. 495-502, 1999.

[32] M.V. Sanchez-Puerta, Y. Cho, J. P. Mower, A. J. Alverson, and J. D. Palmer, "Frequent, phylogenetically local horizontal transfer of the coxl group I intron in flowering plant mitochondria," Molecular Biology and Evolution, vol. 25, no. 8, pp. 1762-1777, 2008.

[33] T. Kubo, S. Nishizawa, A. Sugawara, N. Itchoda, A. Estiati, and T. Mikami, "The complete nucleotide sequence of the mitochondrial genome of sugar beet (Beta vulgaris L.) reveals a novel gene for tRNA ${ }^{\text {Cys }}$ (GCA)," Nucleic Acids Research, vol. 28, no. 13, pp. 2571-2576, 2000.

[34] P. McDermott, V. Connolly, and T. A. Kavanagh, "The mitochondrial genome of a cytoplasmic male sterile line of perennial ryegrass (Lolium perenne L.) contains an integrated linear plasmid-like element," Theoretical and Applied Genetics, vol. 117, no. 3, pp. 459-470, 2008.

[35] A. O. Richardson and J. D. Palmer, "Horizontal gene transfer in plants," Journal of Experimental Botany, vol. 58, no. 1, pp. $1-9,2007$.

[36] P. S. Schnable and R. P. Wise, "The molecular basis of cytoplasmic male sterility and fertility restoration," Trends in Plant Science, vol. 3, no. 5, pp. 175-180, 1998.

[37] M. Satoh, T. Kubo, and T. Mikami, "The Owen mitochondrial genome in sugar beet (Beta vulgaris L.): possible mechanisms of extensive rearrangements and the origin of the mitotype-unique regions," Theoretical and Applied Genetics, vol. 113, no. 3, pp. 477-484, 2006.

[38] G. Bartoszewski, P. Gawronski, M. Szklarczyk, H. Verbakel, and M. J. Havey, "A one-megabase physical map provides insights on gene organization in the enormous mitochondrial genome of cucumber," Genome, vol. 52, no. 4, pp. 299307, 2009.

[39] J. W. Lilly and M. J. Havey, "Small, repetitive DNAs contribute significantly to the expanded mitochondrial genome of cucumber," Genetics, vol. 159, no. 1, pp. 317-328, 2001.

[40] T. Hazle and L. Bonen, "Comparative analysis of sequences preceding protein-coding mitochondrial genes in flowering plants," Molecular Biology and Evolution, vol. 24, no. 5, pp. 1101-1112, 2007.

[41] J. P. Mower, P. Touzet, J. S. Gummow, L. F. Delph, and J. D. Palmer, "Extensive variation in synonymous substitution rates in mitochondrial genes of seed plants," BMC Evolutionary Biology, vol. 7, article 135, 2007.

[42] D. B. Sloan, B. Oxelman, A. Rautenberg, and D. R. Taylor, "Phylogenetic analysis of mitochondrial substitution rate variation in the angiosperm tribe Sileneae," BMC Evolutionary Biology, vol. 9, no. 1, article 260, 2009.

[43] C. M.-R. Fauron, M. Havlik, and R. I. S. Brettell, "The mitochondrial genome organization of a maize fertile cmsT revertant line is generated through recombination between two sets of repeats," Genetics, vol. 124, no. 2, pp. 423-428, 1990.

[44] S. Nishizawa, T. Mikami, and T. Kubo, "Mitochondrial DNA phylogeny of cultivated and wild beets: relationships among cytoplasmic male-sterility-inducing and nonsterilizing cytoplasms," Genetics, vol. 177, no. 3, pp. 1703-1712, 2007.

[45] M. Woloszynska, J. Kieleczawa, M. Ornatowska, M. Wozniak, and H. Janska, "The origin and maintenance of the small repeat in the bean mitochondrial genome," Molecular Genetics and Genomics, vol. 265, no. 5, pp. 865-872, 2001.
[46] M. P. Arrieta-Montiel, V. Shedge, J. Davila, A. C. Christensen, and S. A. Mackenzie, "Diversity of the arabidopsis mitochondrial genome occurs via nuclear-controlled recombination activity," Genetics, vol. 183, no. 4, pp. 1261-1268, 2009.

[47] M. Klein, U. Eckert-Ossenkopp, I. Schmiedeberg, et al., "Physical mapping of the mitochondrial genome of Arabidopsis thaliana by cosmid and YAC clones," The Plant Journal, vol. 6, no. 3, pp. 447-455, 1994.

[48] S. Gabay-Laughnan and K. J. Newton, "Mitochondrial mutations in maize," Maydica, vol. 50, no. 3-4, pp. 349-359, 2005.

[49] G. Bartoszewski, M. J. Havey, A. Ziolkowska, M. Długosz, and S. Malepszy, "The selection of mosaic (MSC) phenotype after passage of cucumber (Cucumis sativus L.) through cell culture-a method to obtain plant mitochondrial mutants," Journal of Applied Genetics, vol. 48, no. 1, pp. 1-9, 2007.

[50] G. Vidal, M. Ribas-Carbo, M. Garmier, et al., "Lack of respiratory chain complex I impairs alternative oxidase engagement and modulates redox signaling during elicitorinduced cell death in tobacco," Plant Cell, vol. 19, no. 2, pp. 640-655, 2007.

[51] W. Sakamoto, H. Kondo, M. Murata, and F. Motoyoshi, "Altered mitochondrial gene expression in a maternal distorted leaf mutant of Arabidopsis induced by chloroplast mutator," Plant Cell, vol. 8, no. 8, pp. 1377-1390, 1996.

[52] R. V. Abdelnoor, R. Yule, A. Elo, A. C. Christensen, G. MeyerGauen, and S. A. Mackenzie, "Substoichiometric shifting in the plant mitochondrial genome is influenced by a gene homologous to MutS," Proceedings of the National Academy of Sciences of the United States of America, vol. 100, no. 10, pp. 5968-5973, 2003.

[53] V. Shedge, M. Arrieta-Montiel, A. C. Christensen, and S. A. Mackenzie, "Plant mitochondrial recombination surveillance requires unusual RecA and MutS homologs," Plant Cell, vol. 19, no. 4, pp. 1251-1264, 2007.

[54] V. Zaegel, B. Guermann, M. Le Ret, et al., "The plant-specific ssDNA binding protein OSB1 is involved in the stoichiometric transmission of mitochondrial DNA in Arabidopsis," Plant Cell, vol. 18, no. 12, pp. 3548-3563, 2006.

[55] M. Odahara, H. Kuroiwa, T. Kuroiwa, and Y. Sekine, "Suppression of repeat-mediated gross mitochondrial genome rearrangements by RecA in the moss physcomitrella patens," Plant Cell, vol. 21, no. 4, pp. 1182-1194, 2009.

[56] A. P. Sandhu, R. V. Abdelnoor, and S. A. Mackenzie, "Transgenic induction of mitochondrial rearrangements for cytoplasmic male sterility in crop plants," Proceedings of the National Academy of Sciences of the United States of America, vol. 104, no. 6, pp. 1766-1770, 2007.

[57] E. V. Kuzmin, D. N. Duvick, and K. J. Newton, "A mitochondrial mutator system in maize," Plant Physiology, vol. 137, no. 2, pp. 779-789, 2005.

[58] I. D. Small, P. G. Issac, and C. J. Leaver, "Stoichiometric differences in DNA molecules containing the atpA gene suggest mechanisms for the generation of mitochondrial genome diversity in maize," The EMBO Journal, vol. 6, no. 4, pp. 865-869, 1987.

[59] I. Small, R. Suffolk, and C. J. Leaver, "Evolution of plant mitochondrial genomes via substoichiometric intermediates," Cell, vol. 58, no. 1, pp. 69-76, 1989.

[60] B. Kmiec, M. Woloszynska, and H. Janska, "Heteroplasmy as a common state of mitochondrial genetic information in plants and animals," Current Genetics, vol. 50, no. 3, pp. 149159, 2006. 
[61] J. Forner, A. Hölzle, C. Jonietz, et al., "Mitochondrial mRNA polymorphisms in different Arabidopsis accessions," Plant Physiology, vol. 148, no. 2, pp. 1106-1116, 2008.

[62] H. Janska, R. Sarria, M. Woloszynska, M. Arrieta-Montiel, and S. A. Mackenzie, "Stoichiometric shifts in the common bean mitochondrial genome leading to male sterility and spontaneous reversion to fertility," Plant Cell, vol. 10, no. 7, pp. 1163-1180, 1998.

[63] K. Kitagawa, S. Takumi, and C. Nakamura, "Selective transcription and post-transcriptional processing of the heteroplasmic mitochondrial orf156 copies in the nucleuscytoplasm hybrids of wheat," Plant Molecular Biology, vol. 53, no. 5, pp. 609-619, 2003.

[64] L. Cao, H. Shitara, M. Sugimoto, J.-I. Hayashi, K. Abe, and H. Yonekawa, "New evidence confirms that the mitochondrial bottleneck is generated without reduction of mitochondrial DNA content in early primordial germ cells of mice," PLoS Genetics, vol. 5, no. 12, Article ID e1000756, 2009.

[65] M. Woloszynska, "Heteroplasmy and stoichiometric complexity of plant mitochondrial genomes-though this be madness, yet there's method in't," Journal of Experimental Botany, vol. 61, no. 3, pp. 657-671, 2010.

[66] M. Woloszynska and D. Trojanowski, "Counting mtDNA molecules in Phaseolus vulgaris: sublimons are constantly produced by recombination via short repeats and undergo rigorous selection during substoichiometric shifting," Plant Molecular Biology, vol. 70, no. 5, pp. 511-521, 2009.

[67] C. M. Barr, M. Neiman, and D. R. Taylor, "Inheritance and recombination of mitochondrial genomes in plants, fungi and animals," New Phytologist, vol. 168, no. 1, pp. 39-50, 2005.

[68] S. A. Pearl, M. E. Welch, and D. E. McCauley, "Mitochondrial heteroplasmy and paternal leakage in natural populations of Silene vulgaris, a gynodioecious plant," Molecular Biology and Evolution, vol. 26, no. 3, pp. 537-545, 2009.

[69] A. Kanazawa, N. Tsutsumi, and A. Hirai, "Reversible changes in the composition of the population of mtDNAs during dedifferentiation and regeneration in tobacco," Genetics, vol. 138, no. 3, pp. 865-870, 1994.

[70] H. Takanashi, T. Ohnishi, M. Mogi, T. Okamoto, S.I. Arimura, and N. Tsutsumi, "Studies of mitochondrial morphology and DNA amount in the rice egg cell," Current Genetics, vol. 56, no. 1, pp. 33-41, 2010.

[71] E. Ducos, P. Touzet, and M. Boutry, "The male sterile G cytoplasm of wild beet displays modified mitochondrial respiratory complexes," The Plant Journal, vol. 26, no. 2, pp. 171-180, 2001.

[72] Y. Kawanishi, H. Shinada, M. Matsunaga, Y. Masaki, T. Mikami, and T. Kubo, "A new source of cytoplasmic male sterility found in wild beet and its relationship to other CMS types," Genome, vol. 53, no. 4, pp. 251-256, 2010.

[73] F. Budar and R. Berthomé, "Cytoplasmic male sterilities and mitochondrial gene mutations in land plants," in Plant Mitochondria, D. C. Logan, Ed., pp. 278-307, Blackwell, Oxford, UK, 2007.

[74] R. E. Dewey, C. S. Levings III, and D. H. Timothy, "Novel recombinations in the maize mitochondrial genome produce a unique transcriptional unit in the texas male-sterile cytoplasm," Cell, vol. 44, no. 3, pp. 439-449, 1986.

[75] H. T. Nivison, C. A. Sutton, R. K. Wilson, and M. R. Hanson, "Sequencing, processing, and localization of the petunia CMS-associated mitochondrial protein," The Plant Journal, vol. 5, no. 5, pp. 613-623, 1994.
[76] K. Ohtani, H. Yamamoto, and K. Akimitsu, "Sensitivity to Alternaria alternata toxin in citrus because of altered mitochondrial RNA processing," Proceedings of the National Academy of Sciences of the United States of America, vol. 99, no. 4, pp. 2439-2444, 2002.

[77] J. R. Marienfeld, M. Unseld, P. Brandt, and A. Brennicke, "Mosaic open reading frames in the Arabidopsis thaliana mitochondrial genome," Biological Chemistry, vol. 378, no. 8, pp. 859-862, 1997.

[78] S. Holec, H. Lange, J. Canaday, and D. Gagliardi, "Coping with cryptic and defective transcripts in plant mitochondria," Biochimica et Biophysica Acta, vol. 1779, no. 9, pp. 566-573, 2008.

[79] M. R. Hanson and S. Bentolila, "Interactions of mitochondrial and nuclear genes that affect male gametophyte development," Plant Cell, vol. 16, supplement, pp. S154S169, 2004.

[80] Z. Wang, Y. Zou, X. Li, et al., "Cytoplasmic male sterility of rice with Boro II cytoplasm is caused by a cytotoxic peptide and is restored by two related PPR motif genes via distinct modes of mRNA silencing," Plant Cell, vol. 18, no. 3, pp. 676687, 2006.

[81] T. Kubo and T. Mikami, "A duplicated sequence in sugarbeet mitochondrial transcripts is differentially edited: analysis of orfB and its derivative orf324 mRNAs," Biochimica et Biophysica Acta, vol. 1307, no. 3, pp. 259-262, 1996.

[82] M. Satoh, T. Kubo, S. Nishizawa, A. Estiati, N. Itchoda, and T. Mikami, "The cytoplasmic male-sterile type and normal type mitochondrial genomes of sugar beet share the same complement of genes of known function but differ in the content of expressed ORFs," Molecular Genetics and Genomics, vol. 272, no. 3, pp. 247-256, 2004.

[83] M. P. Yamamoto, T. Kubo, and T. Mikami, “The 5'-leader sequence of sugar beet mitochondrial atp6 encodes a novel polypeptide that is characteristic of Owen cytoplasmic male sterility," Molecular Genetics and Genomics, vol. 273, no. 4, pp. 342-349, 2005.

[84] M. M. Robison and D. J. Wolyn, "Petaloid-type cms in carrot is not associated with expression of atp 8 (orfB)," Theoretical and Applied Genetics, vol. 112, no. 8, pp. 1496-1502, 2006.

[85] M. M. Robison and D. J. Wolyn, "A $60 \mathrm{kDa}$ COX1 protein in mitochondria of carrot irrespective of the presence of Cterminal extensions in the cox1 reading frames," Molecular Genetics and Genomics, vol. 275, no. 1, pp. 68-73, 2006.

[86] G. Perrotta, J. M. Grienenberger, and J. M. Gualberto, "Plant mitochondrial rps2 genes code for proteins with a C-terminal extension that is processed," Plant Molecular Biology, vol. 50, no. 3, pp. 523-533, 2002.

[87] J. Bailey-Serres, D. K. Hanson, T. D. Fox, and C. J. Leaver, "Mitochondrial genome rearrangement leads to extension and relocation of the cytochrome $c$ oxidase subunit I gene in sorghum," Cell, vol. 47, no. 4, pp. 567-576, 1986.

[88] B. Lu and M. R. Hanson, "A single homogeneous form of ATP6 protein accumulates in Petunia mitochondria despite the presence of differentially edited atp6 transcripts," Plant Cell, vol. 6, no. 12, pp. 1955-1968, 1994.

[89] S. Krishnasamy, R. A. Grant, and C. A. Makaroff, "Subunit 6 of the $\mathrm{F}_{\mathrm{o}}$-ATP synthase complex from cytoplasmic male-sterile radish: RNA editing and $\mathrm{NH}_{2}$-terminal protein sequencing," Plant Molecular Biology, vol. 24, no. 1, pp. 129141, 1994.

[90] K. Kitazaki, Y. Nomoto, A. Aoshima, T. Mikami, and T. Kubo, "A mitochondrial gene involved in cytochrome $c$ maturation 
$(\mathrm{cm} C)$ is expressed as a precursor with a long $\mathrm{NH}_{2}$-terminal extension in sugar beet," Journal of Plant Physiology, vol. 166, no. 7, pp. 775-780, 2009.

[91] X. Zeng, W. Neupert, and A. Tzagoloff, "The metalloprotease encoded by ATP23 has a dual function in processing and assembly of subunit 6 of mitochondrial ATPase," Molecular Biology of the Cell, vol. 18, no. 2, pp. 617-626, 2007.

[92] C. Osman, C. Wilmes, T. Tatsuta, and T. Langer, "Prohibitins interact genetically with Atp23, a novel processing peptidase and chaperone for the $\mathrm{F}_{1} \mathrm{~F}_{\mathrm{O}}$-ATP synthase," Molecular Biology of the Cell, vol. 18, no. 2, pp. 627-635, 2007.

[93] N. Kubo, B. Salomon, T. Komatsuda, R. Von Bothmer, and K. Kadowaki, "Structural and distributional variation of mitochondrial rps2 genes in the tribe Triticeae (Poaceae)," Theoretical and Applied Genetics, vol. 110, no. 6, pp. 9951002, 2005.

[94] X. Zeng, R. Kucharczyk, J.-P. Di Rago, and A. Tzagoloff, "The leader peptide of yeast Atp6p is required for efficient interaction with the Atp9p ring of the mitochondrial ATPase," Journal of Biological Chemistry, vol. 282, no. 50, pp. 3616736176, 2007.

[95] H. Janska, "ATP-dependent proteases in plant mitochondria: what do we know about them today?" Physiologia Plantarum, vol. 123, no. 4, pp. 399-405, 2005.

[96] M. Gibala, M. Kicia, W. Sakamoto, et al., "The lack of mitochondrial AtFtsH4 protease alters Arabidopsis leaf morphology at the late stage of rosette development under shortday photoperiod," The Plant Journal, vol. 59, no. 5, pp. 685699, 2009.

[97] S. Rigas, G. Daras, M. Laxa, et al., "Role of Lon1 protease in post-germinative growth and maintenance of mitochondrial function in Arabidopsis thaliana," New Phytologist, vol. 181, no. 3, pp. 588-600, 2009.

[98] A. R. Abad, B. J. Mehrtens, and S. A. Mackenzie, "Specific expression in reproductive tissues and fate of a mitochondrial sterility-associated protein in cytoplasmic male-sterile bean," Plant Cell, vol. 7, no. 3, pp. 271-286, 1995.

[99] K. Leonhard, B. Guiard, G. Pellecchia, A. Tzagoloff, W. Neupert, and T. Langer, "Membrane protein degradation by AAA proteases in mitochondria: extraction of substrates from either membrane surface," Molecular Cell, vol. 5, no. 4, pp. 629-638, 2000.

[100] E. Itabashi, T. Kazama, and K. Toriyama, "Characterization of cytoplasmic male sterility of rice with Lead Rice cytoplasm in comparison with that with Chinsurah Boro II cytoplasm," Plant Cell Reports, vol. 28, no. 2, pp. 233-239, 2009.

[101] M. P. Yamamoto, H. Shinada, Y. Onodera, C. Komaki, T. Mikami, and T. Kubo, "A male sterility-associated mitochondrial protein in wild beets causes pollen disruption in transgenic plants," The Plant Journal, vol. 54, no. 6, pp. 10271036, 2008.

[102] F. Chaumont, B. Bernier, R. Buxant, M. E. Williams, C. S. Levings III, and M. Boutry, "Targeting the maize $T$ urf13 product into tobacco mitochondria confers methomyl sensitivity to mitochondrial respiration," Proceedings of the National Academy of Sciences of the United States of America, vol. 92, no. 4, pp. 1167-1171, 1995.

[103] S. He, A. R. Abad, S. B. Gelvin, and S. A. Mackenzie, "A cytoplasmic male sterility-associated mitochondrial protein causes pollen disruption in transgenic tobacco," Proceedings of the National Academy of Sciences of the United States of America, vol. 93, no. 21, pp. 11763-11768, 1996.

[104] D. H. Kim, J. G. Kang, and B.-D. Kim, "Isolation and characterization of the cytoplasmic male sterility-associated orf456 gene of chili pepper (Capsicum annuum L.)," Plant Molecular Biology, vol. 63, no. 4, pp. 519-532, 2007.

[105] N. R. Nizampatnam, H. Doodhi, Y. Kalinati Narasimhan, S. Mulpuri, and D. K. Viswanathaswamy, "Expression of sunflower cytoplasmic male sterility-associated open reading frame, orfH522 induces male sterility in transgenic tobacco plants," Planta, vol. 229, no. 4, pp. 987-1001, 2009.

[106] Y. Duroc, S. Hiard, N. Vrielynck, S. Ragu, and F. Budar, "The Ogura sterility-inducing protein forms a large complex without interfering with the oxidative phosphorylation components in rapeseed mitochondria," Plant Molecular Biology, vol. 70, no. 1-2, pp. 123-137, 2009.

[107] D. M. Rhoads, B. Brunner-Neuenschwander, C. S. Levings III, and J. N. Siedow, "Cross-linking and disulfide bond formation of introduced cysteine residues suggest a modified model for the tertiary structure of URF13 in the poreforming oligomers," Archives of Biochemistry and Biophysics, vol. 354 , no. 1, pp. 158-164, 1998. 

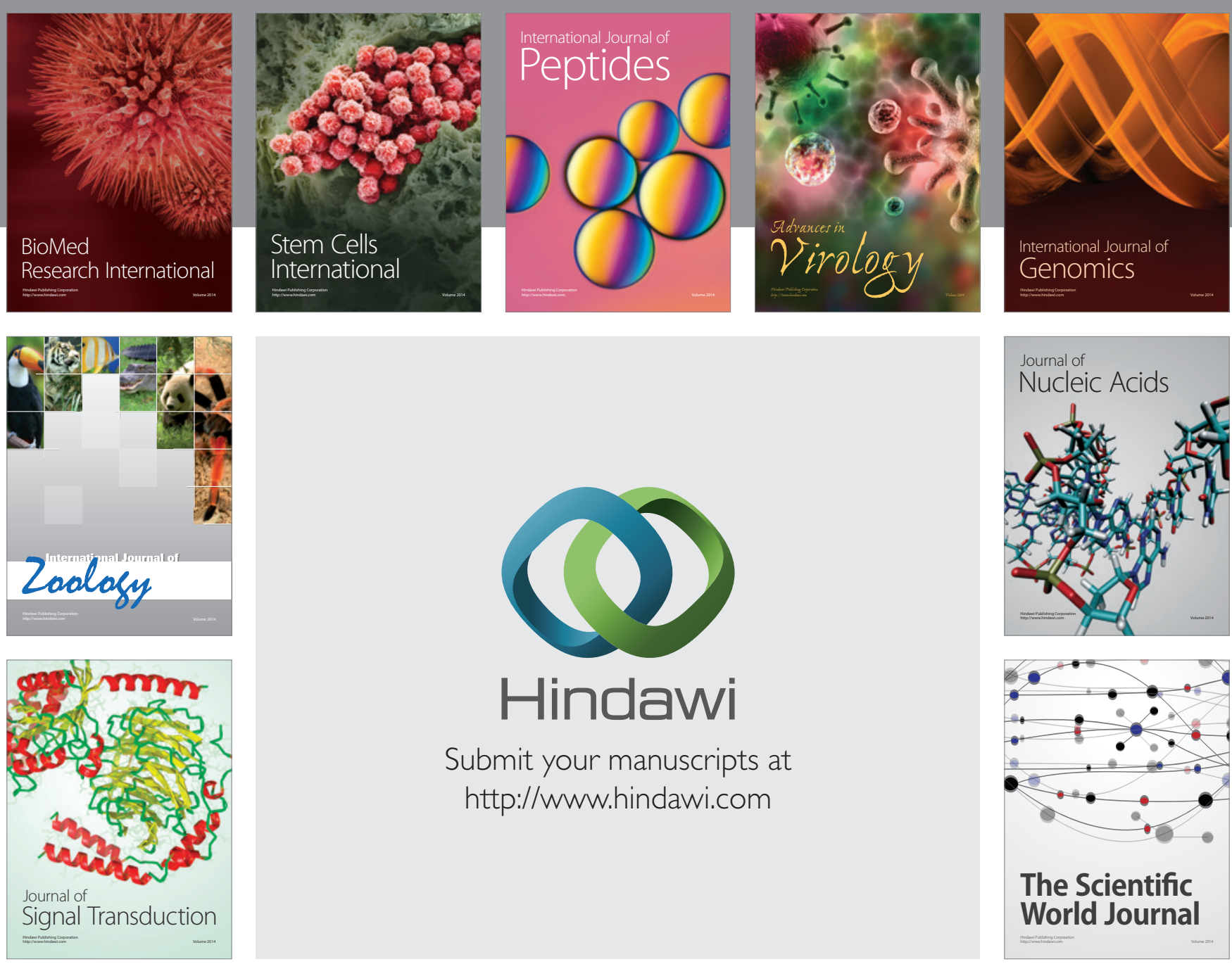

Submit your manuscripts at

http://www.hindawi.com
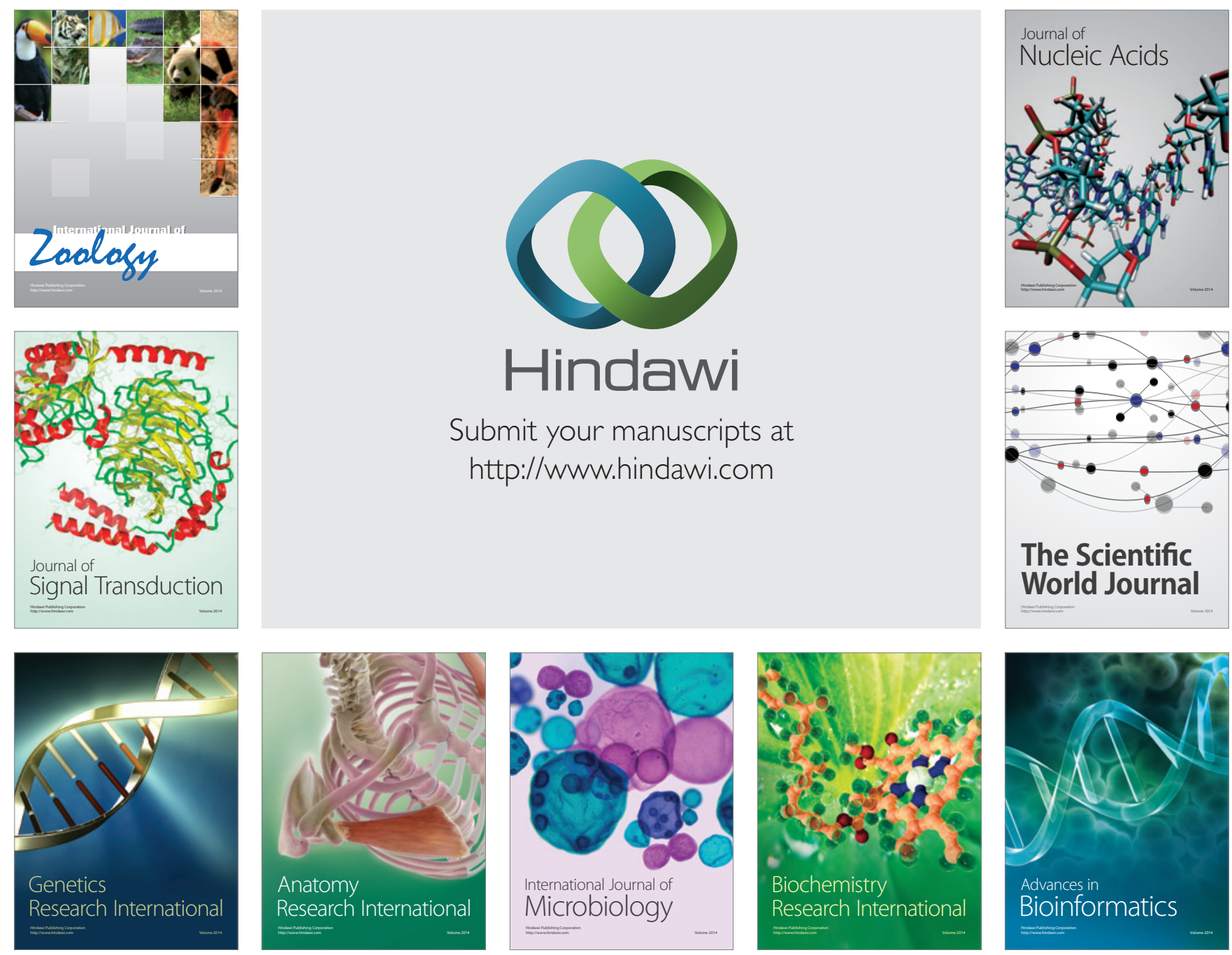

The Scientific World Journal
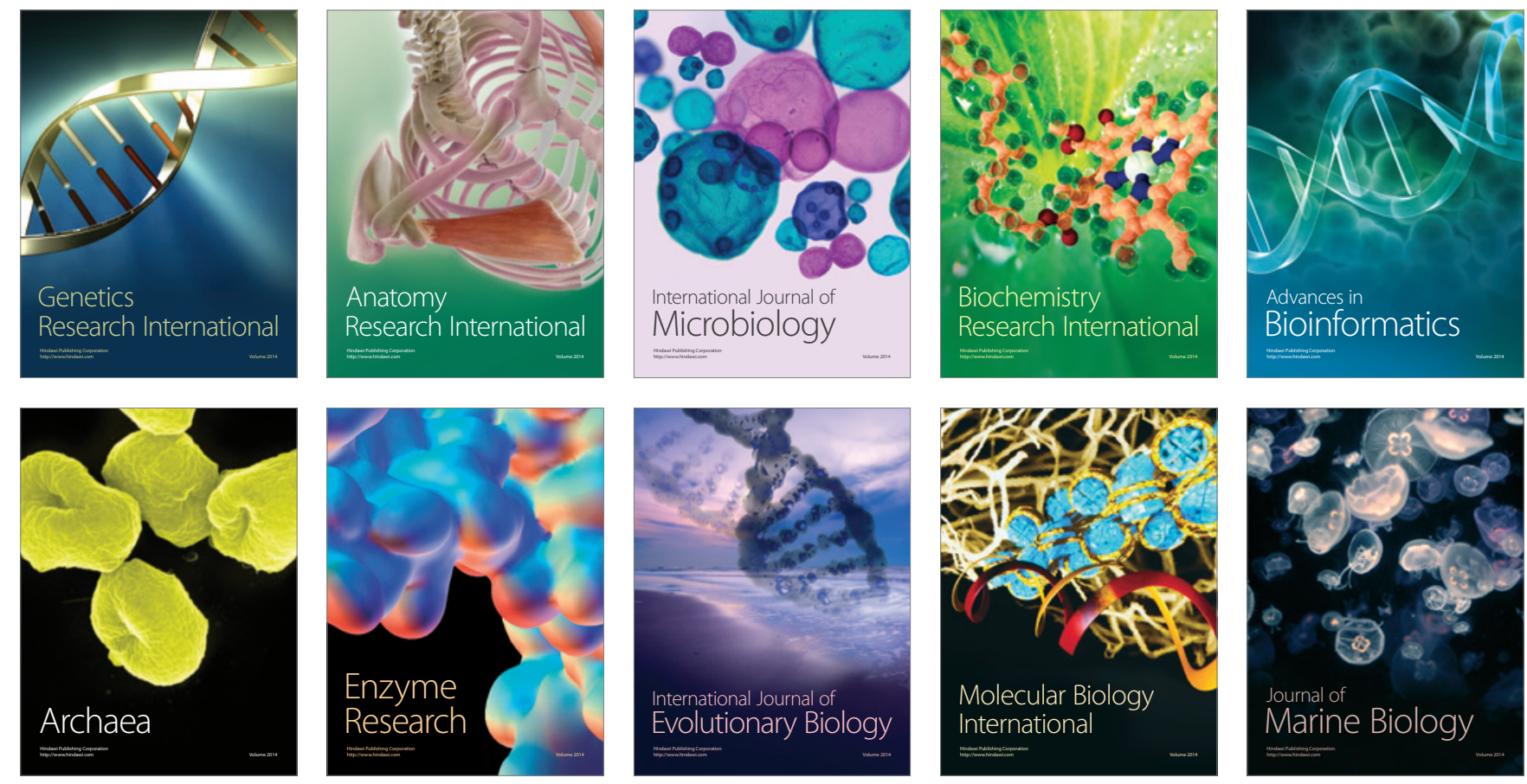